\title{
A Novel Algorithm of DS Evidence Fusion Based on Closeness Degree
}

\author{
Hongxuan Lei ${ }^{+}$, Yan Xue \\ School of computer science and technology, Taizhou University, Taizhou, Jiangsu 225300, China
}

\begin{abstract}
Based on the concept of the closeness degree of fuzzy set, this paper proposes the closeness degree of evidence to measure the similarity between evidences, the closeness matrix and credibility degree are defined by closeness degree of evidence, the weight coefficient of each evidence is obtained by normalizing the credibility degree of evidence, then a novel algorithm of DS evidence fusion is obtained. Some numerical examples and comparisons of this paper demonstrate the effectiveness of the algorithm.
\end{abstract}

Keywords: closeness degree, information fusion, conflict evidence, weight, target recognition

\section{Introduction}

DS evidence theory is a theory of dealing with imprecise reasoning proposed by Dempster and Shafer, it has a strong ability to deal with uncertain information, and has a great deal of advantages: the required conditions are weaker than Bayesian probability theory; there is the ability to express "unknown" and "uncertain" directly [1]. Therefore, it is widely used in information fusion and uncertain reasoning of artificial intelligence [2].

In fact, in the process of information fusion, due to unreliability, uncertainty and conflict of sensors, the efficient fusion of multi-source uncertain information has become a difficult problem. Shafer [3] initially used normalization factor $k$ to express the conflict measurement between two evidences, however, a subsequent study shows that $k$ can't effectively measure the degree of conflict between evidences. Many researchers have proposed many ways to express conflicts, such as Liu [4] proposed to measure the Pignistic probability distance of two evidences. Jousselme et al. [5] proposed evidence distance to indicate the difference between evidences, which is often used to indicate the conflict between evidences [6]; Jiang et al. [7] used $k$ and Jousselme's evidence distance to jointly represent evidence conflict. Murphy [8] proposed a weighted fusion algorithm of average combination of evidences; Deng et al. [9] introduced a distance function of evidence body to calculate the degree of support of each evidence in the system by other evidence, normalized the support degree to obtain the credibility of each evidence as the weight of evidence; Wang et al. [10] proposed a method of evidence combination based on credibility degree and false degree (EC-CF for short). Deng and Wang all used Murphy's method to realize information fusion. Based on the above literature, this paper puts forward the concept of closeness degree between evidences, gives a measure method of evidence credibility by using closeness degree, and then calculates the final fusion result by Murphy's method. Numerical simulation shows that this method has good focusing, fast convergence speed and strong target differentiation.

\section{Preliminaries}

DS theory uses set to express proposition. Let $\Omega$ is a set of all possible values of variable $x$, and the elements in $\Omega$ are mutually exclusive, then it is called the discernment frame of $x$, propositions in the field are represented by subsets of $\Omega$.

\footnotetext{
+ Corresponding author. Tel.: +15161003372, 0523- 80769276.
}

E-mail address: hongxuan_lei@163.com 
Definition 1.[2] Let $m: 2^{\Omega} \rightarrow[0,1]$ be a function, and it be a number $m \in[0,1]$, which satisfies the following

$$
m(\phi)=0 \text { and } \sum_{A \subseteq \Omega} m(A)=1
$$

Then $m$ is called the basic probability assignment (BPA), $m(A)$ is called the basic probability number of proposition $A$, and which is regarded as the reliability accurately assigned to $A$. When $m(A)>0, A$ is called a focal element of $\Omega$.

Definition 2. [2] (Dempster combination rule) Assume that $m_{1}$ and $m_{2}$ are two BPAs, the corresponding focal elements are $A_{1}, A_{2}, \cdots, A_{k}$ and $B_{1}, B_{2}, \cdots, B_{l}, m$ is a new BPA by combining $m_{1}$ and $m_{2}$. The Dempster combination rules are as follows:

$$
\left\{\begin{array}{l}
m(\phi)=0 \\
m(A)=\frac{1}{1-k} \sum_{A_{i} \cap B_{j}=A} m_{1}\left(A_{i}\right) m_{2}\left(B_{j}\right)
\end{array}\right.
$$

Where $k=\sum_{A_{i} \wedge B_{j}=\phi} m_{1}\left(A_{i}\right) m_{2}\left(B_{j}\right)$ is called the conflict coefficient. Combination rule can't be used when $k=1$.

From the formula of $k$, we can get $1-k=\sum_{A_{i} \cap B_{j} \neq \phi} m_{1}\left(A_{i}\right) m_{2}\left(B_{j}\right)$.

By definition 2, let $m_{1}, m_{2}, \cdots, m_{n}$ be $n$ piece of BPAs, then the new evidence $m$ after their combination can be expressed as

$$
\left\{\begin{array}{l}
m(\phi)=0 \\
m(A)=\frac{1}{1-k} \sum_{\cap A_{i}=A} \prod_{1 \leq i \leq n} m_{i}\left(A_{i}\right)
\end{array}\right.
$$

Where $k=\sum_{n A_{i}=\phi} \prod_{1 \leq i \leq n} m_{i}\left(A_{i}\right)$ is the conflict coefficient.

\section{Evidence fusion Algorithm Based on Closeness Degree}

Definition 3. Let $\Omega=\left\{\theta_{1}, \theta_{2}, \cdots, \theta_{n}\right\}$ be a discernment frame, $m_{1}$ and $m_{2}$ are two BPAs, the closeness degree of $m_{1}$ and $m_{2}$ is defined as:

$$
C\left(m_{1}, m_{2}\right)=\frac{\sum_{i=1}^{2^{n}}\left(m_{1}\left(A_{i}\right) \wedge m_{2}\left(A_{i}\right)\right)}{\sum_{i=1}^{2^{n}}\left(m_{1}\left(A_{i}\right) \vee m_{2}\left(A_{i}\right)\right)}, A_{i} \in 2^{\Omega}
$$

Evidently, the following are established:

1. $C\left(m_{1}, m_{2}\right)=C\left(m_{2}, m_{1}\right)$;

2. $0 \leq C\left(m_{1}, m_{2}\right) \leq 1$;

3. $m_{1}=m_{2} \Leftrightarrow C\left(m_{2}, m_{1}\right)=1$;

4. $C\left(m_{1}, m_{2}\right)=0 \Leftrightarrow\left(\cup A_{i}\right) \cap\left(\cup A_{j}\right)=\phi, A_{i}, A_{j}$ are focal elements of $m_{1}, m_{2}$, respectively.

Example 1. Suppose the discernment frame is $\Omega=\left\{\theta_{1}, \theta_{2}, \theta_{3}\right\}$, there are two BPAs which are defined as follows:

then

$$
\begin{aligned}
& m_{1}\left(\left\{\theta_{1}\right\}\right)=0.85, m_{1}\left(\left\{\theta_{1}, \theta_{3}\right\}\right)=0.1, m_{1}(\Omega)=0.05 \\
& m_{2}\left(\left\{\theta_{2}\right\}\right)=0.85, m_{2}\left(\left\{\theta_{3}\right\}\right)=0.05, m_{2}\left(\left\{\theta_{2}, \theta_{3}\right\}\right)=0.05, m_{2}(\Omega)=0.05
\end{aligned}
$$

$$
C\left(m_{1}, m_{2}\right)=0.026
$$

In the above example, the closeness degree of $m_{1}$ and $m_{2} C\left(m_{1}, m_{2}\right)=0.026$, it shows that there is a great conflict between $m_{1}$ and $m_{2}$. At this time, the conflict coefficient $k$ is 0.8925 by the formula (2), it shows that the two evidences are highly conflicting.

Definition 4. (closeness matrix) Let $\Omega$ be the discernment frame, $m_{i}(i=1, \cdots, n)$ be $n$ pieces of BPAs, then the closeness matrix among $n$ evidences based on closeness degree is defined as 


$$
C M=\left[\begin{array}{cccc}
1 & C\left(m_{1}, m_{2}\right) & \cdots & C\left(m_{1}, m_{n}\right) \\
C\left(m_{2}, m_{1}\right) & 1 & \cdots & C\left(m_{2}, m_{n}\right) \\
\cdots & \ldots & \cdots & \cdots \\
C\left(m_{n}, m_{1}\right) & C\left(m_{n}, m_{2}\right) & \cdots & 1
\end{array}\right]
$$

Because $C\left(m_{i}, m_{i}\right)=1(i=1, \cdots, n)$, so the element on the main diagonal of matrix $C M$ is 1 .

The closer the two pieces of evidence are, the more they support each other, so, the credibility degree $\operatorname{Crd}_{i}(i=1, \cdots, n)$ of $m_{i}(i=1, \cdots, n)$ can be measured by its closeness to other BPAs, $\operatorname{Crd}_{i}(i=1, \cdots, n)$ can be expressed as

$$
C r d_{i}=\frac{1}{n-1} \sum_{\substack{j=1 \\ j \neq i}}^{n} C\left(m_{i}, m_{j}\right), i=1, \cdots, n
$$

The credibility degree of $m_{1}, m_{2}, \cdots, m_{n}$ can form a credibility vector

$$
C R D=\left(\mathrm{Crd}_{1}, \mathrm{Crd}_{2}, \cdots, \mathrm{Crd}_{n}\right)^{T}
$$

In particular, when $\mathrm{Crd}_{i}=0$, take $C r d_{i}=\varepsilon, \varepsilon>0$ is any small positive number, according to the specific problem, $\varepsilon$ can take a very small value, thus, the credibility vector is

$$
C R D=\left(\mathrm{Crd}_{1}, \cdots, C r d_{i-1}, \varepsilon, C r d_{i+1}, \cdots C r d_{n}\right)^{T}
$$

The weight coefficient of each BPA after normalization according to formula (7) or $\left(7^{\prime}\right)$ is as follows:

$$
\omega_{i}=\frac{\mathrm{Crd}_{i}}{\sum_{i=1}^{n} C r d_{i}}, i=1,2, \cdots, n
$$

To sum up, the fusion algorithms of $n$ evidences can be expressed as follows:

1. Input $n$ pieces of BPA $m_{1}, m_{2}, \cdots, m_{n}$;

2. Compute closeness matrix $\boldsymbol{C M}$ from formula (4) and (5);

3. Compute the credibility degree of each BPA according to formula (6): $\operatorname{Crd}_{i}, i=1,2, \cdots, n$;

4. Compute the credibility vector according to formula (7) or ( $\left.7^{\prime}\right)$ :

$$
C R D=\left(\mathrm{Crd}_{1}, \mathrm{Crd}_{2}, \cdots, C r d_{n}\right)^{T} \text { or } C R D=\left(\mathrm{Crd}_{1}, \cdots, C r d_{i-1}, \varepsilon, C r d_{i+1}, \cdots C r d_{n}\right)^{T} \text {; }
$$

5. Compute the weight coefficient $\omega_{i}(i=1,2, \cdots, n)$ according to formula (8), get the weight coefficient vector $\omega=\left(\omega_{1}, \omega_{2}, \cdots, \omega_{n}\right)$;

6. According to $\omega$, weight average of $n$ pieces of BPA, obtain a new BPA $\bar{m}$;

7. Combine $\bar{m} n$-1 times by the formula (3), obtain final BPA $m$ of $n$ pieces of evidence.

Example 2. Let $\Omega=\left\{\theta_{1}, \theta_{2}, \theta_{3}\right\}$ be the discernment frame, and

$$
\begin{aligned}
& m_{1}\left(\left\{\theta_{1}\right\}\right)=0.85, m_{1}\left(\left\{\theta_{1}, \theta_{3}\right\}\right)=0.1, m_{1}\left(\left\{\theta_{1}, \theta_{2}, \theta_{3}\right\}\right)=0.05 \\
& m_{2}\left(\left\{\theta_{2}\right\}\right)=0.85, m_{2}\left(\left\{\theta_{3}\right\}\right)=0.05, m_{2}\left(\left\{\theta_{2}, \theta_{3}\right\}\right)=0.05, m_{2}\left(\left\{\theta_{1}, \theta_{2}, \theta_{3}\right\}\right)=0.05 \\
& m_{3}\left(\left\{\theta_{1}\right\}\right)=0.9, m_{3}\left(\left\{\theta_{3}\right\}\right)=0.1
\end{aligned}
$$

Compute the BPA $m$ after fusion of $m_{1}, m_{2}$ and $m_{3}$ as follows.

Compute the closeness degree of three BPAs from formula (4):

$$
C\left(m_{1}, m_{2}\right)=0.026, C\left(m_{1}, m_{3}\right)=0.739, C\left(m_{2}, m_{3}\right)=0.026
$$

Get the closeness matrix from formula (5)

$$
C M=\left[\begin{array}{ccc}
1 & 0.026 & 0.739 \\
0.026 & 1 & 0.026 \\
0.739 & 0.026 & 1
\end{array}\right]
$$

Obtain the credibility vector from formula (6) and (7):

$$
C R D=(0.3825,0.026,0.3825)^{T}
$$

Obtain the weight coefficient vector of $m_{1}, m_{2}, m_{3}$ from formula (8):

$$
\omega=\left(\omega_{1}, \omega_{2}, \omega_{3}\right)=(0.4836,0.0328,0.4836)^{T}
$$


Weight average of three pieces of BPA according to $\omega$ :

$$
\begin{aligned}
& \bar{m}\left(\left\{\theta_{1}\right\}\right)=0.84630, \bar{m}\left(\left\{\theta_{2}\right\}\right)=0.02788, \bar{m}\left(\left\{\theta_{3}\right\}\right)=0.05000, \\
& \bar{m}\left(\left\{\theta_{1}, \theta_{3}\right\}\right)=0.04836, \bar{m}\left(\left\{\theta_{2}, \theta_{3}\right\}\right)=0.00164, \bar{m}\left(\left\{\theta_{1}, \theta_{2}, \theta_{3}\right\}\right)=0.02582
\end{aligned}
$$

Combine $\bar{m}$ two times by the formula (3), have

$$
\begin{aligned}
& m\left(\left\{\theta_{1}\right\}\right)=0.875, m\left(\left\{\theta_{2}\right\}\right)=0.001, m\left(\left\{\theta_{3}\right\}\right)=0.047, \\
& m\left(\left\{\theta_{1}, \theta_{3}\right\}\right)=0.050, m\left(\left\{\theta_{2}, \theta_{3}\right\}\right)=0.000, m\left(\left\{\theta_{1}, \theta_{2}, \theta_{3}\right\}\right)=0.027
\end{aligned}
$$

\section{Numerical Example}

In order to illustrate the performance of this algorithm, we still list the examples in [10].

Example 3. Suppose the discernment frame is $\Omega=\left\{\theta_{1}, \theta_{2}, \theta_{3}\right\}$, there are five BPAs which are defined as follows:

$$
\begin{aligned}
& m_{1}\left(\left\{\theta_{1}\right\}\right)=0.5, m_{1}\left(\left\{\theta_{2}\right\}\right)=0.2, m_{1}\left(\left\{\theta_{3}\right\}\right)=0.3 \\
& m_{2}\left(\left\{\theta_{1}\right\}\right)=0, m_{2}\left(\left\{\theta_{2}\right\}\right)=0.85, m_{2}\left(\left\{\theta_{3}\right\}\right)=0.15 \\
& m_{3}\left(\left\{\theta_{1}\right\}\right)=0.6, m_{3}\left(\left\{\theta_{2}\right\}\right)=0.1, m_{3}\left(\left\{\theta_{3}\right\}\right)=0.3 \\
& m_{4}\left(\left\{\theta_{1}\right\}\right)=0.65, m_{4}\left(\left\{\theta_{2}\right\}\right)=0.25, m_{4}\left(\left\{\theta_{3}\right\}\right)=0.1 \\
& m_{5}\left(\left\{\theta_{1}\right\}\right)=0.55, m_{5}\left(\left\{\theta_{2}\right\}\right)=0.1, m_{5}\left(\left\{\theta_{3}\right\}\right)=0.35
\end{aligned}
$$

We obtain the closeness matrix from formula (4) and (5) as follows:

$$
C M=\left[\begin{array}{ccccc}
1 & 0.212 & 0.818 & 0.667 & 0.818 \\
0.212 & 1 & 0.143 & 0.212 & 0.143 \\
0.818 & 0.143 & 1 & 0.667 & 0.905 \\
0.667 & 0.212 & 0.667 & 1 & 0.6 \\
0.818 & 0.143 & 0.905 & 0.6 & 1
\end{array}\right]
$$

We construct the credibility degree vector according to formula (6) as follows:

$$
C R D=\left(\mathrm{Crd}_{1}, \mathrm{Crd}_{2}, \mathrm{Crd}_{3}, \mathrm{Crd}_{4}, \mathrm{Crd}_{5}\right)^{T}=(0.629,0.178,0.633,0.537,0.617)^{T}
$$

We obtain the weight coefficient vector from formula (7) and (8) as follows:

$$
\omega=(0.242,0.069,0.244,0.207,0.238)^{T}
$$

We obtain the weighted average BPA after weighting average five pieces of BPA:

$$
\bar{m}\left(\left\{\theta_{1}\right\}\right)=0.533, \bar{m}\left(\left\{\theta_{2}\right\}\right)=0.207, \bar{m}\left(\left\{\theta_{3}\right\}\right)=0.260
$$

Combine $\bar{m}$ four times by the formula (3), have

$$
m\left(\left\{\theta_{1}\right\}\right)=0.964, m\left(\left\{\theta_{2}\right\}\right)=0.009, m\left(\left\{\theta_{3}\right\}\right)=0.027
$$

In the following, through the above example, comparing the methods of Dempster combination, Murphy's method, Deng's method, method of EC-CF, and the methods of this paper, we can fuse the BPAs, as shown in Table 1.

Table 1: Comparison between other evidence combination methods and the methods in this paper

\begin{tabular}{lllll}
\hline Various methods & \multicolumn{1}{c}{$\oplus_{i=1}^{2} m_{i}$} & \multicolumn{1}{c}{$\oplus_{i=1}^{3} m_{i}$} & $\oplus_{i=1}^{4} m_{i}$ & $\oplus_{i=1}^{5} m_{i}$ \\
\hline \multirow{2}{*}{ Dempster's method } & $m\left(\left\{\theta_{1}\right\}\right)=0$ & $m\left(\left\{\theta_{1}\right\}\right)=0$ & $m\left(\left\{\theta_{1}\right\}\right)=0$ & $m\left(\left\{\theta_{1}\right\}\right)=0$ \\
& $m\left(\left\{\theta_{2}\right\}\right)=0.791$ & $m\left(\left\{\theta_{2}\right\}\right)=0.557$ & $m\left(\left\{\theta_{2}\right\}\right)=0.759$ & $m\left(\left\{\theta_{2}\right\}\right)=0.474$ \\
& $m\left(\left\{\theta_{3}\right\}\right)=0.209$ & $m\left(\left\{\theta_{3}\right\}\right)=0.443$ & $m\left(\left\{\theta_{3}\right\}\right)=0.241$ & $m\left(\left\{\theta_{3}\right\}\right)=0.526$ \\
\hline \multirow{2}{*}{ Murphy's method } & $m\left(\left\{\theta_{1}\right\}\right)=0.161$ & $m\left(\left\{\theta_{1}\right\}\right)=0.407$ & $m\left(\left\{\theta_{1}\right\}\right)=0.683$ & $m\left(\left\{\theta_{1}\right\}\right)=0.865$ \\
& $m\left(\left\{\theta_{2}\right\}\right)=0.709$ & $m\left(\left\{\theta_{2}\right\}\right)=0.464$ & $m\left(\left\{\theta_{2}\right\}\right)=0.279$ & $m\left(\left\{\theta_{2}\right\}\right)=0.102$ \\
& $m\left(\left\{\theta_{3}\right\}\right)=0.130$ & $m\left(\left\{\theta_{3}\right\}\right)=0.129$ & $m\left(\left\{\theta_{3}\right\}\right)=0.038$ & $m\left(\left\{\theta_{3}\right\}\right)=0.033$ \\
\hline \multirow{2}{*}{ Deng's method } & $m\left(\left\{\theta_{1}\right\}\right)=0.161$ & $m\left(\left\{\theta_{1}\right\}\right)=0.617$ & $m\left(\left\{\theta_{1}\right\}\right)=0.870$ & $m\left(\left\{\theta_{1}\right\}\right)=0.952$ \\
& $m\left(\left\{\theta_{2}\right\}\right)=0.709$ & $m\left(\left\{\theta_{2}\right\}\right)=0.232$ & $m\left(\left\{\theta_{2}\right\}\right)=0.095$ & $m\left(\left\{\theta_{2}\right\}\right)=0.020$ \\
& $m\left(\left\{\theta_{3}\right\}\right)=0.130$ & $m\left(\left\{\theta_{3}\right\}\right)=0.151$ & $m\left(\left\{\theta_{3}\right\}\right)=0.035$ & $m\left(\left\{\theta_{3}\right\}\right)=0.028$ \\
\hline Method of EC-CF & $m\left(\left\{\theta_{1}\right\}\right)=0.161$ & $m\left(\left\{\theta_{1}\right\}\right)=0.665$ & $m\left(\left\{\theta_{1}\right\}\right)=0.905$ & $m\left(\left\{\theta_{1}\right\}\right)=0.964$
\end{tabular}




\begin{tabular}{lllll} 
& $m\left(\left\{\theta_{2}\right\}\right)=0.709$ & $m\left(\left\{\theta_{2}\right\}\right)=0.181$ & $m\left(\left\{\theta_{2}\right\}\right)=0.063$ & $m\left(\left\{\theta_{2}\right\}\right)=0.011$ \\
& $m\left(\left\{\theta_{3}\right\}\right)=0.130$ & $m\left(\left\{\theta_{3}\right\}\right)=0.154$ & $m\left(\left\{\theta_{3}\right\}\right)=0.032$ & $m\left(\left\{\theta_{3}\right\}\right)=0.025$ \\
\hline \multirow{3}{*}{ Proposed method } & $m\left(\left\{\theta_{1}\right\}\right)=0.161$ & $m\left(\left\{\theta_{1}\right\}\right)=0.733$ & $m\left(\left\{\theta_{1}\right\}\right)=0.916$ & $m\left(\left\{\theta_{1}\right\}\right)=0.964$ \\
& $m\left(\left\{\theta_{2}\right\}\right)=0.709$ & $m\left(\left\{\theta_{2}\right\}\right)=0.115$ & $m\left(\left\{\theta_{2}\right\}\right)=0.050$ & $m\left(\left\{\theta_{2}\right\}\right)=0.009$ \\
& $m\left(\left\{\theta_{3}\right\}\right)=0.130$ & $m\left(\left\{\theta_{3}\right\}\right)=0.152$ & $m\left(\left\{\theta_{3}\right\}\right)=0.034$ & $m\left(\left\{\theta_{3}\right\}\right)=0.027$ \\
\hline
\end{tabular}

It can be seen from the above table that Dempster's method can't recognize $\left\{\theta_{1}\right\}$, this is due to $m_{2}\left(\left\{\theta_{1}\right\}\right)=0$, the conflict between the second BPA and the other four BPAs is too great, which results in "one vote veto". As can be seen from the five BPAs, except for the second evidence, the other four evidence greatly support $\left\{\theta_{1}\right\}$, from the comparison results, Murphy's method needs to collect four pieces of evidence to identify target $\left\{\theta_{1}\right\}\left(m\left(\left\{\theta_{1}\right\}\right)=0.683\right)$, and the convergence speed is slow, because this method uses the mean value as the weight coefficient. The methods of Deng and EC-CF needs to collect three pieces of evidence to identify $\left\{\theta_{1}\right\}$. The methods of this paper also needs three pieces of evidence to identify target $\left\{\theta_{1}\right\}$ completely, and the convergence rate is faster than the method of Deng and EC-CF. With more and more evidences, the support of target $\left\{\theta_{1}\right\}$ increases rapidly, and the discriminative power between targets increases further, which shows that proposed method has better properties of convergence and focus, which is very beneficial to the final decision-making.

\section{Conclusions}

Firstly, this paper puts forward the concept of the closeness degree of evidence to measure the similarity between evidences. The smaller the closeness degree of two evidences is, the greater the conflict will be, conversely, the smaller the conflict will be. When the two evidences are completely consistent, the closeness degree of evidence is 1 , indicating that there is no conflict between the two evidences. Secondly, the closeness matrix and the evidence credibility degree are defined by using the closeness degree, and the weight coefficient of each evidence is obtained by normalizing the evidence credibility degree. Finally, $n$ pieces of BPA are weighted and averaged according to the weight coefficient of each evidence, and the BPA obtained by weighted average is fused $n-1$ times according to Murphy's method to get the final fusion.

The numerical simulation results show that the algorithm is simple in thought, small in calculation, fast in convergence and strong in focusing performance. The conflict between evidences has always been an open issue, how to find a reasonable and effective method is our next research content.

\section{Acknowledgements}

This work is partially supported by Taizhou University high level talents research initiation fund (Program No. TZXY2017QDJJ011).

\section{References}

[1] D.Q.Han, Y.Yang, D.C.Han. The research progress of DS evidence theory and related problems. Control and decision making,2014,29(1):1-11.

[2] W.Jiang, X.Y.Deng . Information modeling and application of D-S theory. Beijing: Science Press,2018.

[3] G. Shafer. A mathematical theory of evidence. Princeton University Press, 1976.

[4] W.R.Liu. Analyzing the degree of conflict among belief functions. Artificial intelligence,2006,170(11):909-924.

[5] A.L.Jousselme, D.Grenier, E.Boss. A new distance between two bodies of evidence. Information fusion,2001, 2(2):91-101.

[6] W.Jiang, C. Xie, B.Wei,et al. A modified method for risk evaluation in failure modes and effects analysis of aircraft turbine rotor blades. Advances in mechanical engineering,2016,8(4):1-16.

[7] W.Jiang, J.Y.Peng, Y.Deng. A new representation of evidence conflict. System engineering and electronic technology,2010,32(3):562-565.

[8] C.A.Murphy. Combining belief functions when evidence conflicts. Decision support systems,2000,29(1):1-9.

[9] Y.Deng, W.K.Shi, Z.F.Zhu. An effective combination method of conflict evidence. Journal of infrared and millimeter wave, 2004, 23(1):27-32.

[10] X.D.Wang, Y.F.Song, Z.H.Si. Uncertain information representation and fusion technology.Beijing: Science Press, 2018 . 